\title{
Serum rheumatoid factors in spinal cord injury patients
}

\author{
N V Petrova MD,${ }^{1}$ A M Ponomaryova MD ${ }^{2}$ V A Alyoshkin MD ${ }^{3}$ A T Eliseyev MD,${ }^{4}$ \\ G S Yumashev $\mathrm{MD}^{5}$ \\ ${ }^{1}$ Senior Research Worker, ${ }^{4}$ Director of the Laboratory of Spine and Spinal Cord Injuries, \\ ${ }^{5}$ Head of Department, I M Sechenov Moscow Academy; ${ }^{2}$ Senior Research Worker, \\ ${ }^{3}$ Director of the Laboratory of Biochemistry, GN Gabrichevsky Institute of Epidemiology \\ and Microbiology, Moscow, Russia.
}

The rheumatoid factor (RF) levels of IgM-RF and IgG-RF types in sera from
patients with spinal cord injuries (SCI) and osteochondrosis were investigated by
ELISA. According to the findings in the late stages of the disease, SCI patients
showed $46 \%$ seropositiveness for IgG-RF and $40 \%$ for IgM-RF. Patients with
fresh central nervous system (CNS) injuries were $12 \%$ and $14 \%$ seropositive,
respectively. Patients with osteochondrosis exhibited $33 \%$ seropositiveness for
IgG-RF. The determination of serum RF enables us to identify the systemic
body lesion in the SCI late stages as a rheumatoid disease. These data support
the conclusion that RF determination is a reliable serological indicator of the
severity of a patient's traumatic disease.

Keywords: rheumatoid factors; ELISA; spinal cord injury.

\section{Introduction}

In the late stages of disease many SCI patients exhibit systemic lesions of the visceral organs along with disturbed motor activity. Taking into consideration the severity of a number of symptoms, spinal cord trauma in the advanced stages can be referred to autoimmune disorders. Chronic inflammation and significant immune system disorders in injury ${ }^{1,2}$ suggest involvement of antibodies to autologous immunoglobulins (the RF) in pathological development. ${ }^{3}$ To clarify the role of RF in spinal cord trauma in the late stages, serum RF levels of $\operatorname{IgG}$ and $\operatorname{IgM}$ were investigated, and it was speculated that such studies could be used prognostically to predict the outcome of SCI patients.

\section{Patients and methods}

IgM-RF and IgG-RF serum levels were determined in the patient groups as follows: (1) spinal cord trauma in the late stages (4 or more months after injury); (2) spinal injury in the early stages (within 2 weeks of injury); (3) craniocerebral injury; (4) spinal pathological states (osteochondrosis). RF was determined by ELISA. To create the immunoenzyme system our own and commercial reagents were used: (1) Fc-fragment of human IgG; (2) staphylococcal protein A and horse radish peroxidase conjugate; (3) substrate mixture-orthophenylenediamine and hydrogen peroxide solution; (4) carrier-polystyrene 96-well pans ('Titertek'). The ELISA data were recorded on a Titertek multiscan photometer at $492 \mathrm{~nm}$ wavelength. RF preparations, isolated and purified by affinity chromatography from seropositive sera of rheumatoid arthritis (RA) patients and RA patients' sera, were used as positive controls. IgG and IgM preparations, isolated from normal human sera and from the sera pooled of healthy blood donors, were used as negative controls. Seventy-nine serum samples from patients with CNS injuries and nontraumatic spinal cord diseases, and samples from healthy blood donors were analysed by ELISA. The ratio between optical densities (OD) of the test sample and the diluted (1:50) negative control serum was used as a criterion to estimate the results, according to the formula $\mathrm{OD}(\mathrm{x}) / \mathrm{OD}(-)$. The results 
were compared with the discriminating index determined in analysing sera from healthy blood donors by the formula:

$$
\frac{\sum \mathrm{OD}\left(x_{1}-x_{n}\right) / \mathrm{OD}(-)}{n}+2 \delta
$$

where $\sum \operatorname{ODD}\left(x_{1}-x_{n}\right)=$ the value sum total, $\mathrm{OD}(-)=$ the negative control optical density, $n=$ the number of samples from blood donors. Sera with a staining intensity exceeding the correction factor obtained in analysis of the control group are considered positive. $^{4}$

\section{Results and discussion}

ELISA used in this study enabled us to detect RF of IgG-RF and IgM-RF types in patients' sera (Table I). The RF levels of both types with late stage SCI (group 1) significantly exceeded the levels in patients of other groups. According to the findings, estimated with respect to the discriminating index obtained from the donor sera, trauma patients were $46 \%$ seropositive for IgG-RF and $40 \%$ for IgM-RF. Patients with fresh CNS injuries (groups 2 and 3) showed low RF levels and 12-14\% seropositiveness, double the RF detection frequency in healthy individuals. ${ }^{5}$ An increase of RF levels in patients with osteochondrosis (group 4), 33\% seropositive for IgG-RF,

Table I The results of the determination of rheumatoid factors of $\operatorname{IgG}$ and $\operatorname{IgM}$ classes

No Patient group Seropositive Seropositive for IgG-RF for IgM-RF

\begin{tabular}{|c|c|c|c|}
\hline 1 & $\begin{array}{l}\text { Spinal cord injury } \\
\quad \text { (late stage) } \\
n=47\end{array}$ & $46 \%$ & $40 \%$ \\
\hline 2 & $\begin{array}{l}\text { Recent spinal cord } \\
\text { injury } \\
n=7\end{array}$ & $14 \%$ & - \\
\hline 3 & $\begin{array}{l}\text { Craniocerebral } \\
\text { injury } \\
n=8\end{array}$ & $12 \%$ & - \\
\hline 4 & $\begin{array}{l}\text { Nontraumatic } \\
\text { spinal diseases } \\
n=7\end{array}$ & $33 \%$ & $14 \%$ \\
\hline
\end{tabular}

was recorded. The estimation of seropositiveness for RF, introduced for examination of RA patients, is reported to be reliable as a criterion of disease intensity and indispensable for predicting possible complications. ${ }^{3}$ The RF determination technique used by us in examining SCI patients is quite reasonable as advanced trauma and rheumatic disease (RA, acute rheumatism, reactive arthritis) are similar in many respects. Common symptoms are peripheral joint and spinal lesions, and cardiac disturbances. ${ }^{6}$ In traumatic disease the development of rheumatic symptoms depends on the SCI level and the time interval since the injury. Clinically manifested symptoms of rheumatic complications (massive inflammation, heterotopic ossification, and tissue fibrosis) are found only in advanced trauma. According to our clinical observations, SCI patients (group 1) developed cardiac disturbances and nephrolithiasis, considered by some authors ${ }^{7,8}$ as manifestations of degenerative disorders. Clinically in connective tissue illnesses, particularly in traumatic disease, cardiovascular disturbances are rarely diagnosed, and thus various special examinations (polycardiografy, echocardiografy etc) are required. ${ }^{9}$ Traditional examination procedures used in this study enabled us to detect the following changes in the patients of group 1: pulse rate and arterial pressure lability; tachycardia and bradycardia; and extrasystoles, often regarded as cardiac disturbances. Visceral disorders were associated with increased IgM-RF levels in the sera from the group 1 patients compared to the donor group. Forty percent of group 1 patients were seropositive for IgM-RF. IgM-RF occurrence in patients is prognostically a hazardous factor. ${ }^{10}$ Therefore it may be concluded that in many spinal patients the complicated disease course, manifested by a number of visceral disorders, may be related to significant shifts in the immune system and to IgM-RF accumulation in blood.

As has been reported, ${ }^{10-12}$ the IgG-RF level correlates with rheumatic disease intensity. Since the disease severity in spinal patients is in many respects determined by the intensity of the inflammation, it is clear why in selected laboratory tests the inflam- 
mation rate and IgG-RF level were changing unidirectionally. IgG-RF was reported to be detected in blood after secondary immunisation, and RF formation was preceded by an increase in circulating immune complexes (CIC). ${ }^{13} \mathrm{CIC}$ involvement in inflammatory processes has been validated. ${ }^{6}$ Following the increase in CIC level, IgG type RF is assumed to contribute to the progressive inflammation. ${ }^{11,14}$ The results of CIC level determination in spinal patients were described in our previous paper. ${ }^{15}$ Patients with high CIC values had pressure ulcers and chronic inflammatory disease of the urinary system. These findings indicated a correlation between these symptoms and such indicators as an increase in urine protein and leukocytes, ESR, blood fibrinogen and the IgG-RF level, although IgM-RF values may remain low. Simultaneous increases in IgG-RF and IgM-RF levels correlated with the most serious disease forms.

Of the four groups examined, patients with recent spinal injuries (group 2), and craniocerebral injuries (group 3) exhibited certain similarities in IgM-RF and IgG-RF levels: IgM-RF was not detected and the groups were similar to donors in levels of IgG-RF. The above phenomenon is consistent with the concept that in CNS injuries there are certain dynamics in the immune system responses and only in the late stages of the disease do conditions exist for the formation of autoantibodies to autologous RF immunoglobulins. ${ }^{16}$

Noteworthy is the increase in IgG-RF level in patients with nontraumatic spinal cord disease (group 4), of which 33\% were seropositive. Patients with osteochondroses have a number of similarities with spinal injury patients. On examination, patients in group 4 showed cardiovascular disturbances (right bundle-branch block, bradycardia, arrhythmia, myocardial trophic disturbance), hepatic fibroid degeneration, uterine polyp, urinary system changes, and other visceral disorders. The development of visceral disorders is also connected with involvement of the vegetative nervous system. In osteochondroses and spinal injuries, disradicular conflicts involving the sympathetic nervous system may occur. ${ }^{7}$ The postganglionic fibre compression rate in bone and muscular canals and involvement of the sympathetic chain and other vegetative nervous system formations in patients of groups 1 and 4 may vary, thus evidently accounting for the differences in the clinical manifestations of vegetovascular disorders as well as the immunological shifts. It is significant that, with unidirectional IgG-RF changes in groups 1 and 4 , the RF level varies. In SCI patients, IgG-RF values and disease severity can be assumed to be determined by vegetovascular dystonia occurring against the background of degenerative organ restructuring caused by disturbed central influence.

Certain difficulties in establishing the original cause of RF formation do not hinder the wide use of this index in disease diagnosis. RF occurrence is reported to be related to the complex course of chronic illnesses such as endocarditis, tuberculosis, chronic hepatitis, and typhoid fever. ${ }^{9}$

$\mathrm{RF}$ determination to estimate inflammation intensity may be of particular significance in examining SCI patients, as pain sensation, the most important symptom of joint inflammation and destruction in spinal patients, may be lost. This study demonstrates the expediency of using the criterion of seropositiveness in estimating the severity of spinal cord traumatic disease, selecting the therapy technique, and determining the drug efficacy.

\section{Conclusions}

For SCI patients in the late stages (group 1) seropositiveness was $46 \%$ for IgG-RF and $40 \%$ for IgM-RF. RF detection proved to be a reliable serological test to estimate the severity of the patient's condition.

In the early stages of spinal cord injury (group 2) and of craniocerebral injury (group 3), seropositiveness for IgG-RF was $10-12 \%$, double the RF level in healthy individuals.

Patients with osteochondrosis (group 4) showed a marked increase in IgG-RF: seropositiveness was $33 \%$. This may be related to the constriction of postganglionic fibres in bony and muscular canals.

RF detection in the blood of spinal cord 
injury patients enables us to identify the systemic body lesion in the late stages of SCI as a disease of the rheumatoid group.
The presence of seropositiveness should be taken into account in prescribing the treatment of SCI patients.

\section{References}

1 Detlav IE (1976) Antibrain antibodies in the blood serum and CSF following brain trauma. Zhurnal Nevropatologii i psikhiatrii im. S. S. Korsakova 26(3): 344-348. Voprosy Nejrokhirurgii 5: 32-35.

2 Kogan OG, Knyazhev VA, Belyaev AF (1981) Some parameters of specific humoral immunity in patients with trauma to the spinal cord. Voprosy Kurortologii, Fisyoterapii i Lechebnoj Fiskultury 4: 55-58.

3 Zborobsky AB, Lempert BA, Yugtina ME (1989) Clinical and diagnostic significance of rheumatoid factors of various classes in rheumatoid arthrits. Sovetskaya meditsina 12: 6-9.

4 Ponomaryova AM, Denisova OV, Popkov M Yu, Alyoshkin VA (1989) Identification of IgG rheumatoid factor in reumatoid arthrits by enzyme immunology. Immunologiya 3: 89-91.

5 Palosuo T, Aho K (1983) Technical falsely positive reumatoid factor by ELISA in sera with elevated IgM levels. Med Biol 61: 203-207.

6 Nasonova VA, Astapenko MG (1989) Clinical Rheumatology. Meditsina, Moscow.

7 Leonovich AL (1990) Actual Questions of Neuropathology. Vysshaya shkola, Minsk.

8 Yekutiel M, Brooks ME, Ohry A, Yarom J, Carel R (1989) The prevalence of hypertension ischaemia heart disease and diabetes in traumatic spinal cord injured patients and amputees. Paraplegia 27: 58-62.

9 Tupikin GV, Kogan BM, Kuzmishin LS, Sokolova DA, Redina TA (1984) Diagnosis of cardiovascular disorders in rheumatoid arthritis patients. Reumatologiya 2: 22-25.

10 Pope RM, McDuffy SI (1979) IgG rheumatoid factor. Relationship to seropositive rheumatoid arthritis and absence in seronegative disorders. Arthritis Rheum 22: 988-998.

11 Withrington RH, Teitsson J, Valdimarsson H, Scifert MH (1984) Prospective study of early rheumatoid arthritis. II Association of rheumatoid factor isotypes with fluctuations in disease activity. Ann Rheum Dis 43: $679-685$.

12 Zlabinger GJ, Haberhauer G, Dax K, Menzel EJ, Bröll H (1990) Rheumatoid factor isotypes and circulating immune complexes in rheumatoid arthritis. Clin Exp Immunol 8: 113-119.

13 Malashkhiya Yu A, Geladze MG, Lomdzhariya LD, Mikeladze NA (1982) Cerebrospinal fluid as the immunological barrier of the brain. Immunologia 6: 76-78.

14 Pobedina VG, Geilfand VB (1977) Functional activity of blood leukocytes in traumatic lesion of spinal cord. Zhurnal Voprosy Neirokhirurgii im. N. N. Burdenko iss. 5, 25-28.

15 Yumashev GS, Petrova NV, Eliseyev AT, Alyoshkin VA, Rozina MN (1991) Immune complexes of blood and liquor of patients with trauma of spinal cord. Klinicheskaya Meditsina 3: 56-58.

16 Gannushkina IV (1974) Immunological aspects of brain injury and cerebral vascular disturbances. Meditsina, Moscow: 199

17 Pope RM, Yoshinoya S, McDuffy SI (1981) Detection of immune complexes and their relationship to rheumatoid factor in a variety of autoimmune disorders. Clin Exp Immunol 46: 259-267. 